\title{
Magneto Mechanical Properties of Iron Based MR Fluids
}

\author{
S. Elizabeth Premalatha ${ }^{1,2}$, R. Chokkalingam ${ }^{1}$, M. Mahendran ${ }^{1, *}$ \\ ${ }^{1}$ Smart Materials Lab, Department Physics, Thiagarajar College of Engineering, Madurai, 625015, India \\ ${ }^{2}$ Department of Physics, Sri S. Ramasamy Naidu Memorial College, Sattur, 626203, India
}

\begin{abstract}
The main aim of this article is to prepare MR fluids, composed of iron particles and analyse their flow behaviour in terms of the internal structure, stability and magneto rheological properties. MR fluids are prepared using silicone oil (OKs) mixed with iron powder. To reduce sedimentation, grease is added as stabilizers. The size of the particles is observed by Optical microscope and flow properties are examined by rheometer. Sedimentation is measured by simple observation of changes in boundary position between clear and turbid part of MR fluid placed into glass tube. The various additive percentages can also influence the MR fluid's performances.
\end{abstract}

Keywords Magnetorheological Fluid, Apparent Viscosity, Yield Stress

\section{Introduction}

Using some standard materials, science and technology have made amazing development in the design of electronics and machinery. Such materials have the ability to change their shape or size by adding a little bit of heat or to change from the liquid to a solid when this material is near to a magnet. These materials are called smart materials. Magnetic functional fluids are also regarded as smart materials[1]. Magnetic functional fluids are able to change their rheological properties when it is placed in an external magnetic field[2].Magnetic functional fluid (MF) and Magnetorheological fluid (MRF) are two common types of these fluid. The only difference between these two fluids is the inner particle size. In magnetic functional fluid, the particle size are about $10 \mathrm{~nm}$ in diameter but the particles in magnetorheological are in the order of microns.

Magnetorheological fluid (MRF) are the suspensions of these micron size polarisable particles in a magnetically neutral fluid[3]. Silicone oil or mineral oil is usually used as magnetically neutral fluid. In MR fluid, some small additives are added, which affect the polarization of the particles and also stabilization of the structure of the suspension to resist settling of particles[4].The first development of MR fluid was credited to JACOB Rainbow (1949) at the US National Bureau of Standards[5,6]. MR fluids contain the magnetisable particles, non-magnetisable suspending fluids and additives. MR fluids response to an applied field and have ability to change from a linear viscous fluid with free floe to a semi-solid state. The behaviour of MR fluid can be described by theplastic model under applied external field.

* Corresponding author:

manickam-mahendran@tce.edu (M. Mahendran)

Published online at http://journal.sapub.org/ajps

Copyright (C) 2012 Scientific \& Academic Publishing. All Rights Reserved
When the field is cut off, the MR fluid comes to original state.MR fluid behave like Newton fluid in zero magnetic field. When certain amount of magnet field is applied, the magnetic particles form chain cluster due to dipole-dipole interaction between particles[7-12]. The important characteristics of the magnetically active dispersed phase are particle size, shape, density, particle size distribution, saturation magnetization and coercive field [13] Other than these properties of magnetic particles, the base fluids, surfactants, anticorrosion, additives are important factors that affect the rheological properties, stability and redispersibility of the MR fluid.

MR fluids appear similar to liquid paints and exhibit comparable levels of apparent viscosity $(0.1$ to $1 \mathrm{~Pa}$ at low shear rates), in the "OFF" state[14]. Apparent viscosity MR fluid changes significantly $\left(10^{5}-10^{6}\right)$ times within milliseconds, when the magnetic field is applied[15]. This effect is completely reversible, when the magnetic field is removed. Controlling the intensity of the magnetic field applied in the MR fluid can adjust the apparent viscosity and other rheological properties of the fluid[16]. Particles are held together by magnetic field and the chains of the particles resist to a certain level of shear stress without breaking, which make them behave like a solid.

The materials start to flow and structure breaks when shear stress exceeds a critical value. This critical shear stress value is the 'apparent yield stress' of the material. Within a possible magnetic field strength range MR fluid can exhibit a yield stress of $10-100 \mathrm{kPa}[17]$. The yield stress is very important in many applications. This property depends on volume fraction of magnetic particles and applied magnetic field. The yield stress is also affect by particle distribution. Shear strain occurs in the fluid, when the MR fluid flows and also shear stress distribution develops across the fluid[4]. The viscous flow equations of elementary fluid mechanics help to calculate this shear distribution. 
The MR fluids can be widely used in designing damping devices such as shock absorbers, clutches, surface polishing machine parts and vibration dampers[18-29]. Soft ferro magnetic materials like magnetite and iron are adopted as the MR materials[30-31]. These particles are easily magnetized under magnetic field. Silicone oil, mineral oil, synthetic hydrocarbon compound or other suitable organic liquids are used as carrier liquids.

The frequent problem in MR fluids application is the tendency of the active magnetic particles to aggregate and settle down, that disturbs the homogeneity of MR fluid and could influence its properties. This is because magnetic particles are denser than liquid carrier and under gravity settle and form a hard "cake" which makes them impossible to redisperse. The aim of this work is to prepare magneto rheological fluids with different additive percentage. The particle size is also investigated. Rheological properties of the MR suspensions under applied magnetic strengths are also then investigated. The flow behaviour and viscoelastic properties are measured by the steady state and oscillation experiments respectively. The sedimentation effect of MR fluids is also studied.

\section{Experimental Procedure}

\subsection{MR Fluid Materials}

For the preparation of MR fluid, micro sized powder of iron $(99.5 \% \mathrm{Fe}, 250-300 \mathrm{mesh})$ with average size of $28 \mu \mathrm{m}$ and silicone oil (OKs) as carrier fluid which has stable temperature in the interval $-40^{\circ} \mathrm{C}$ to $250^{\circ} \mathrm{C}$ are used. Also some additives are considered to prevent sedimentation and coagulation as well as to facilitate dispersion of particles. Here grease is used as additives.

Table 1. Components of MR fluid

\begin{tabular}{|c|c|c|c|c|}
\hline Types & $\begin{array}{c}\text { Diameter } \\
\mu \mathrm{m}\end{array}$ & $\begin{array}{c}\text { Concentration } \\
\text { Wt } \%\end{array}$ & Carrier & $\begin{array}{c}\text { Additive } \\
\text { Percentage }\end{array}$ \\
\hline MRF1 & 28 & 39 & Silicon oil & 0.5 \\
\hline MRF2 & 28 & 39 & Silicon oil & 0.25 \\
\hline MRF3 & 28 & 39 & Silicon oil & 0.15 \\
\hline
\end{tabular}

\subsection{Preparation Method}

First, commercially purchased grease is added with silicone oil. Then the dispersion medium is mixed with a mechanical stirrer at $400 \mathrm{rpm}$ in room temperature. To prepare the MR fluid, iron particles with diameter of $28 \mu \mathrm{m}$ are dispersed in silicone oil. Here three kinds of MR fluids are prepared and they are named as MRF1, MRF2, and MRF3. The concentration of iron particles are 39\% (fixed) in all MR fluid (MRF1, MRF2, and MRF3). Their corresponding additives were in three different percentages $(0.5 \%, 0.25 \%$ and $0.15 \%)$. The components of MR fluids are shown in Table1. The sedimentation is measured by visual observation of the position changes of boundary between clear and turbid part of carrier oil. Prepared samples are placed into cylindrical glass beaker (BOROSIL
$50 \mathrm{ml}$ ) for few days. As a result sedimentation ratio(R) is calculated. Sedimentation ratio is simply defined as a proportion between length of clear and turbid part of MR fluid.

\subsection{Characterization}

In order to determine the mean size of iron particles of powder dispersed, the MRF samples were analysed. Diameter of the iron particles are observed with optical scanning microscope (Olympus Microscope-BX 41-TF). MR fluid is also examined through this microscope in $40 \mathrm{X}$ magnification. The microscope photos are taken with the help of Olympus - camediac - 7070 digital camera. Rheological properties of MR suspensions are examined with and without magnetic field using a parallel plate type rheometer (Anton paar MCR 301, Anton paar MCR) at 25. ${ }^{\circ} \mathrm{C}$ The coil current and magnetic field strength using separate control unit and rheometer software are (Rheoplus ) applied.

\section{Results and Discussion}

Figure 1 shows the size of the iron particle observed from Optical scanning microscope with magnification.

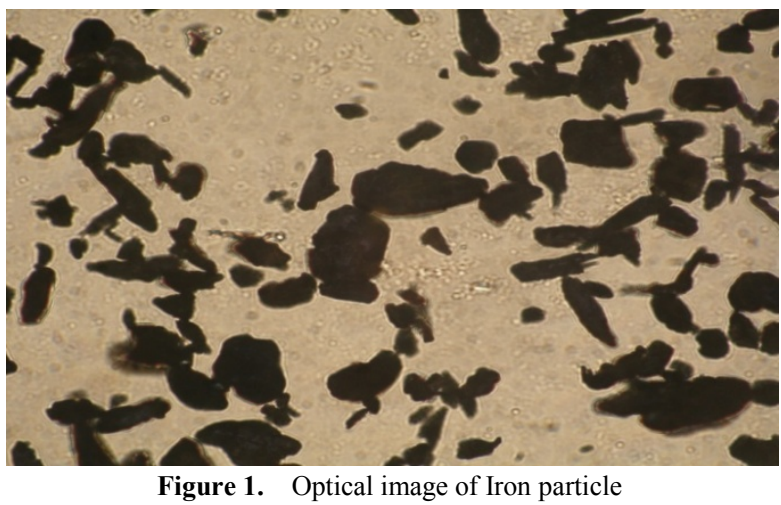

The data analysis refers to more than 100 particles. The mean size of the iron particles was determined about $28 \mu \mathrm{m}$. The figure 2 represents the image of MR fluid by same Optical scanning microscope. In this figure, the iron particles are dispersed in carrier fluid.

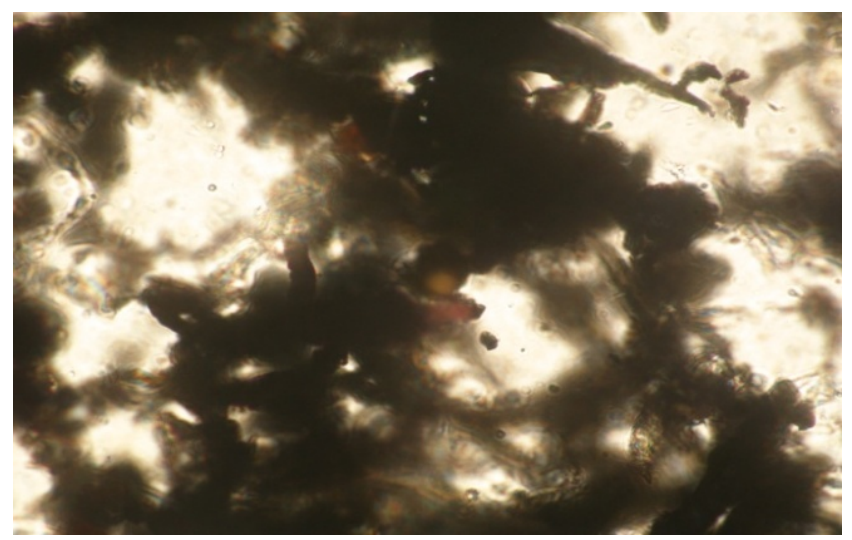

Figure 2. Optical image of MR fluid 
Figure 3 represents the sedimentation ratio of the particles with and without additives. Higher content of magnetic particles has positive impact on the magnetorheological effect. The effect of additives on the stability of the magnetorheological fluid has been investigated exper imentally.

Stability of prepared sample takes different values and it varies only few minutes. It was depend on the content of iron particles and also the type of the oil, which was used.

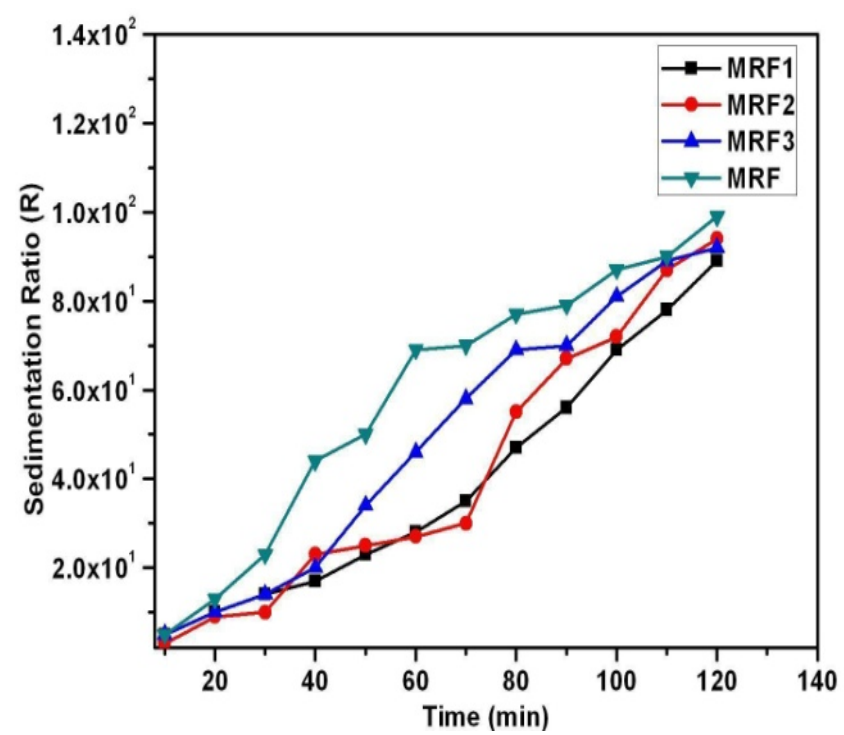

Figure 3. Time vs sedimentation ratio with and without additives

To further improve their stability, additives are added to the MR fluid. MR fluid without additive is named as MRF. Here the particles settle down quickly in the fluid without additive. The variation was observed in the graph due to different percentage of additives. The better stability of the MR fluid is obtained by increasing of the amount of additives[33]. It shows that MRF1 was much better than others.

Figure 4 represent the relationship between the shear stress and shear strain for all three MR fluids.

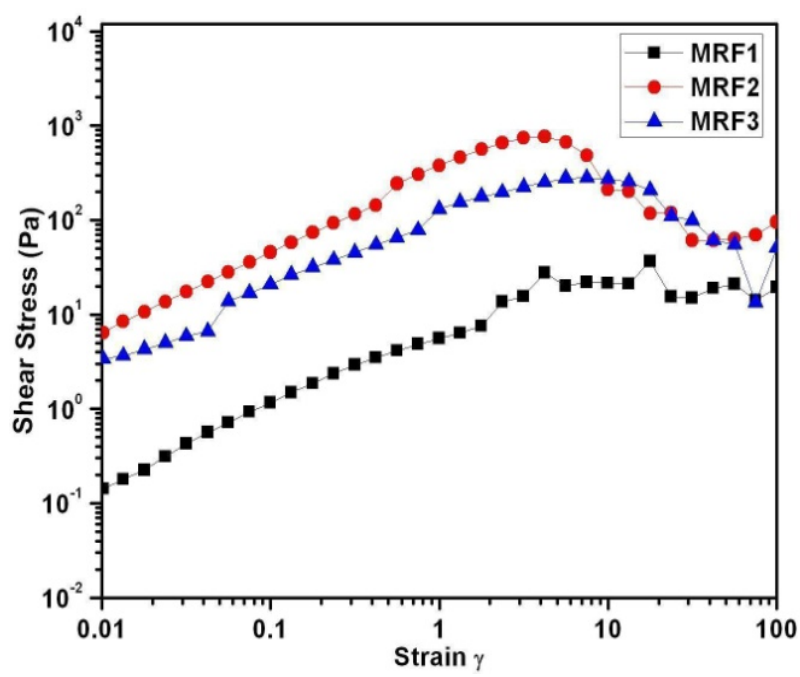

Figure 4. Strain vs shear stress for MRF1,MRF2,MRF3
Typical non-Newtonian fluid behaviour is observed in the absence of magnetic field. The yield stress value is high for MRF3. The stress value increases with strain value up to 10 percentages. After that the structure of MR fluid breaks, rupture takes place. Then the fluid starts to flow and it changes into liquid state from semi solid state. On the other hand, apparent yield stress can be obtained by extrapolating the shear stress values at zero shear strain value. It is assumed to be caused by the remnant magnetization of the magnetic particle[32]. The yield stress value for MRF1, MRF2 and MRF3 are equal to $0.21 \mathrm{~Pa}, 9 \mathrm{~Pa}$ and $7 \mathrm{~Pa}$ respectively. The maximum shear stress value is equal to $800 \mathrm{~Pa}$. MRF2 shows high value of yield stress.

Figure5 represent the viscosity value of all MRfluids with shear rate.

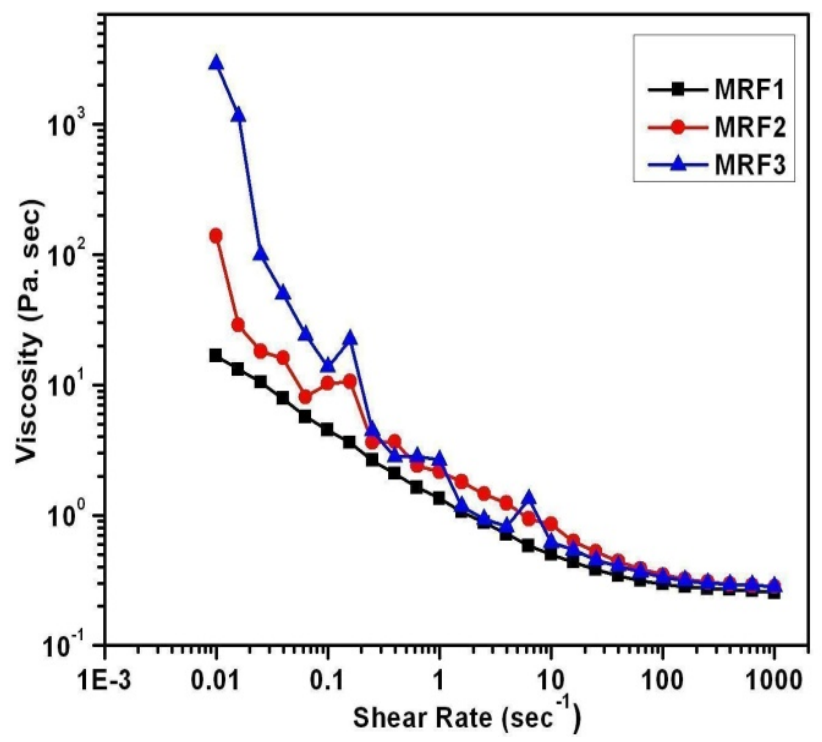

Figure 5. Shear rate vs Viscosity for MRF1, MRF2, MRF3

The viscosity value is measured in Pa sec. Here non Newtonian flow behaviour is observed at zero magnetic field. Here, viscosity decreases with increasing shear rate. At low shear rate, the viscosity of MRF 3 is greater than other. At higher shear rate, the value of all three samples is same. At higher shear rate the value of viscosity is equal to nearly $0.2 \mathrm{~Pa} \mathrm{sec}$. Shear thinning behaviour of the MR suspensions is observed.

Figure $6(\mathrm{a}-\mathrm{c})$ shows the storage modulus and loss modulus (G', G') as a function of frequency with constant magnetic field and without magnetic field for MRF1, MRF2, and MRF3. G' is the measure of the energy stored and recovered per cycle, known as storage modulus. The measure of the energy dissipated or lost as heat per cycle is called as loss modulus (G'). The storage modulus G' is the measurement of the elasticity and loss modulus is the property related to the shear viscosity. Here, LG' and LG" represent storage modulus and loss modulus for MRF1, MRF2, MRF3 in the absence of magnetic field. SG' and SG" represent storage modulus and loss modulus for MRF1, MRF2, and MRF3 in the presence of magnetic field. 


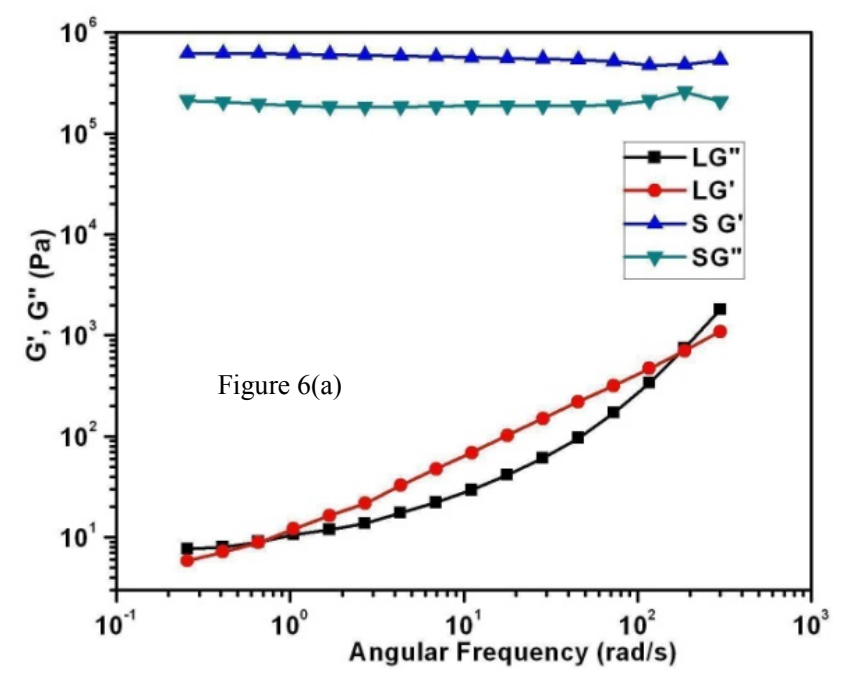

(a)
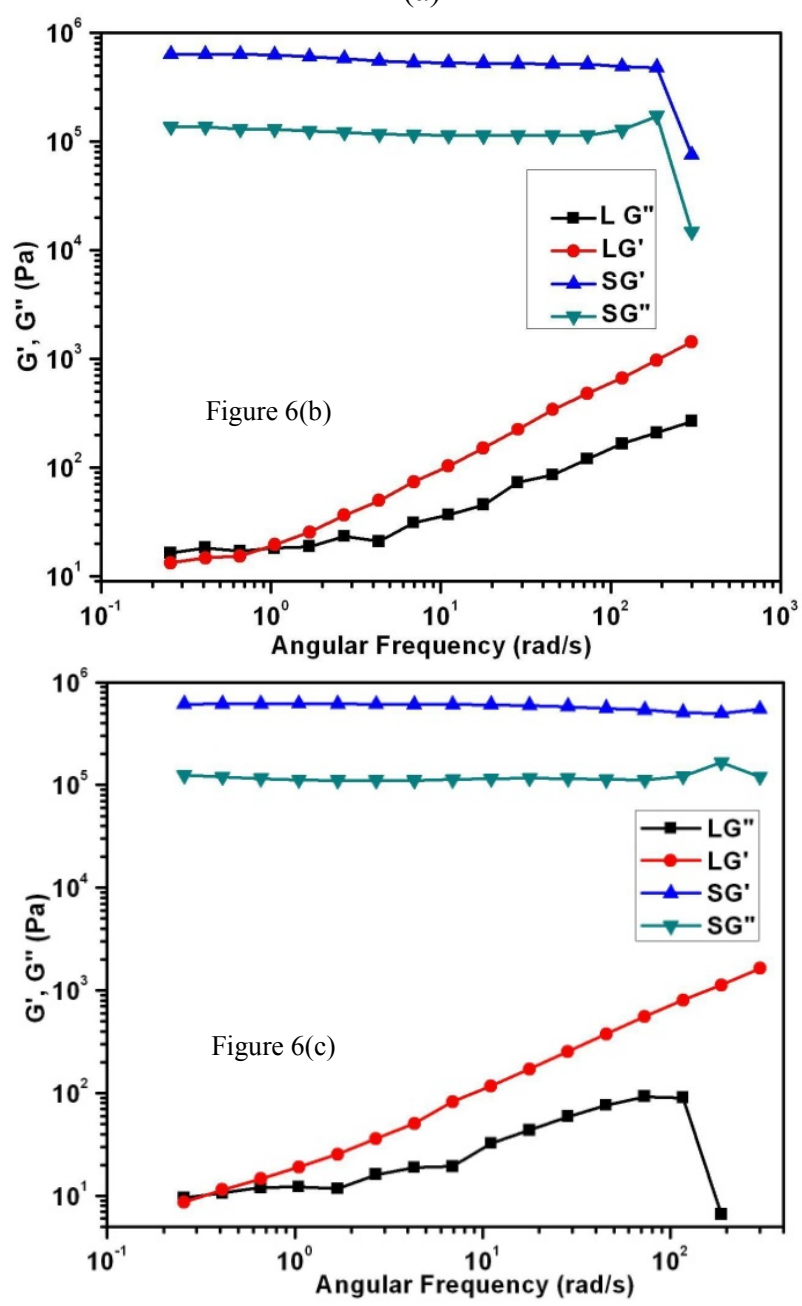

Figure 6 (a-c). Angular frequency versus G' and G" (with and without magnetic field) curve

It is found that, the sample possesses superior solid like structure rather than liquid like structure because both storage and loss modulus do not vary apparently with the growth of frequency at the fixed magnetic strength [34-36]. It is also to be noted that the storage modulus is always higher than loss modulus over the frequency range. It is leading to the elastic property of the MR fluids. Figure 7 represents magnetic field sweep curve of three MR fluids.

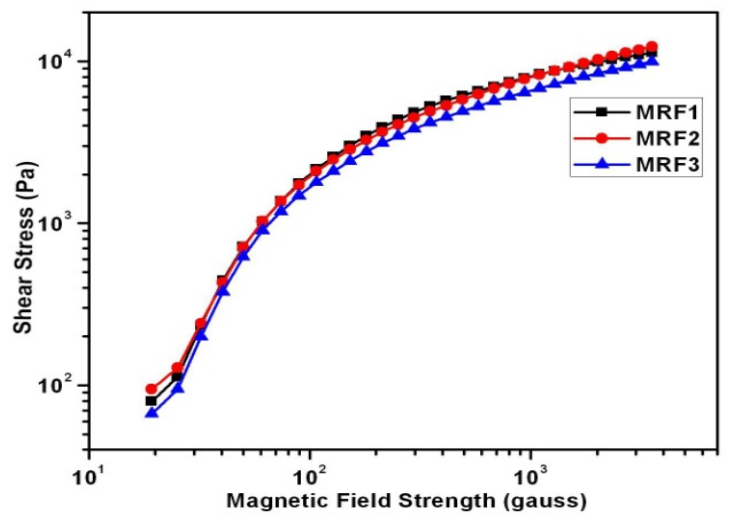

Figure 7. Magnetic field vs shear stress for MRF1, MRF2 and MRF3
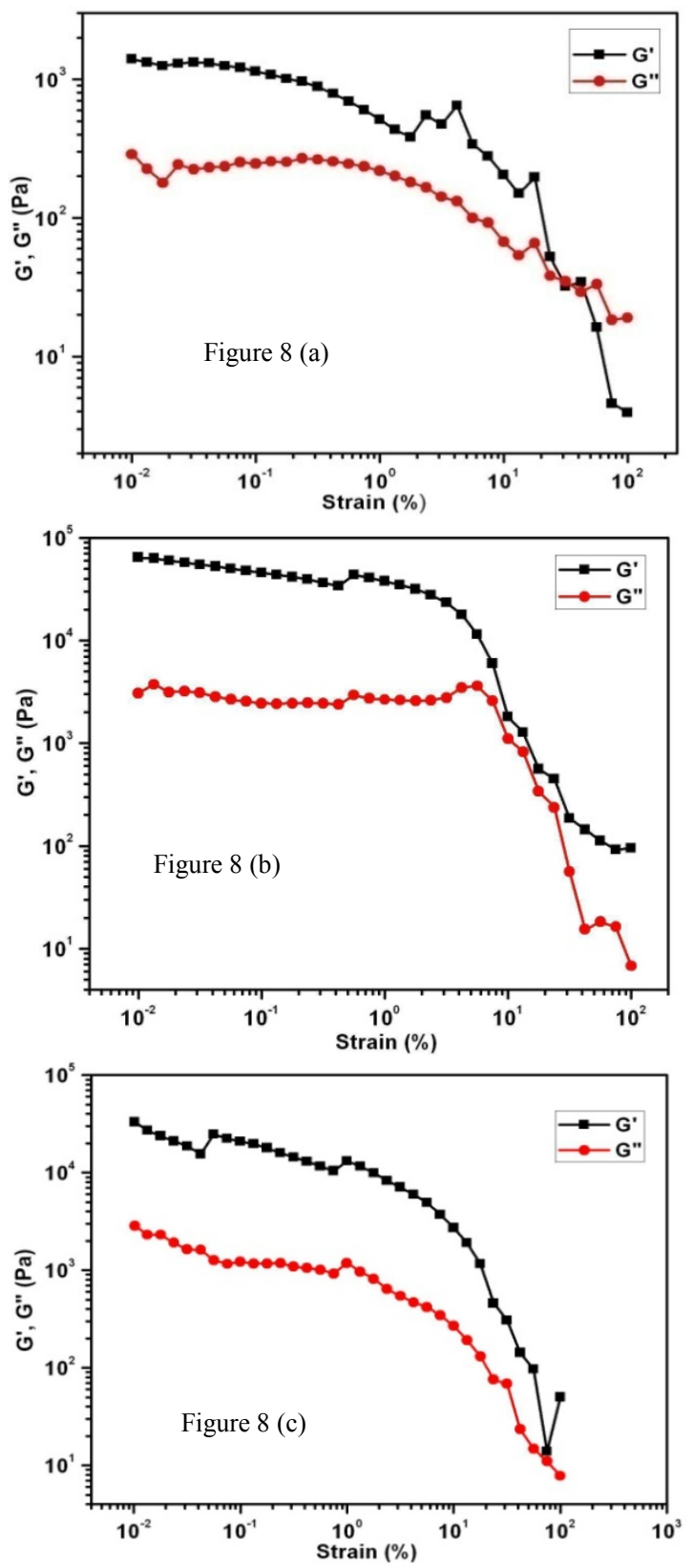

Figure 8 (a-c). Strain versus G' and G' (without magnetic field) curve 
The measurements are taken from 0 to 3600 gauss. The changes in the shear stress with magnetic field are noted. Due to the increased magnetic field strength, short chains sticking to each other via collision grow in the flow field. It is explained that, in the flow direction the arrangement of particles is favourable [37].

It causes affection by the shear deformation, exhibiting shear thinning behaviour. The measurements are conducted only in increasing magnetic field value. The fluid began to be affected by the shear deformation. It exhibits shear thinning behaviour $[38,39]$. Such a short chains stick to each other via collision and grow in the flow field due to the increased magnetic field strength.

Figure 8 (a-c) represents amplitude sweep (without magnetic field) curve. From the amplitude sweep, the storage modulus $\left(G^{\prime}\right)$ values tested without magnetic field, increases sharply, loss modulus (G') also shows the same trend. In the initial range of strain, the storage modulus $\left(G^{\prime}\right)$ show little dependence on the strain, then declined gradually due to the broken chain structure. At the high range of strain, liquid like behaviour will be observed. In the amplitude over this value, the structure of the sample will be irreversibly altered or even completely ruined, while smaller than this value, the structure of the sample will be sustained.

\section{Conclusions}

In this study, the particle size of iron power is confirmed by Optical Scanning Microscope. The magnetic properties of iron particles dispersed in silicone oil were measured. It can be seen that the shear stress increased with shear strain. The yield stress of each MR fluids is obtained. This fluid exhibits non-Newtonian behaviour in the absence of magnetic field. The shear thinning behaviour is observed in shear viscosity data and magnetic sweep data. The storage modulus and loss modulus value are also increased indicating very strong elastic properties. It is also observed that the sedimentation is improved by adding higher percentage of additives.

\section{ACKNOWLEDGEMENTS}

The Authors thanks the Indian Institute of Technology, Madras and Indira Gandhi Centre of Research, Kalpakkam.

\section{REFERENCES}

[1] Min,S.,Cho.,Sung,T.,Lim.,Jang,In.Bb.,Young,J.,Choi., and Myung.S.John.,July2004,EncapsulationofSphericalIron-Parti le With PMMA and its Magnetorhological Particles, IE Transaction on Magnetics, Vol.40,No.4.

[2] Bramantya,M.A.,Motozawa,M.,Sawada,T.,2010,Ultrasonic
Propergatipn Velocity in Magnetic and Magnetorheological fluidsduetoanExternalMagnetorheologicalfiel,J.Phys.Conden s.Matter22(5pp)

[3] Chiriac,H., and Stoian,G.,2010,Influence of particle size distribution on magnetorheological fluid performances,Jou

[4] Tran Hai NAM., and Kyoung Kwan AHN.,2009,New Approch to Designing aN MR Brake using a Small Steel Roller and MR fluid,Journal of Mechanical Science and Technology 23,1911-1923.

[5] Rabiiow, J., 1948, The magnetic Fluid Cluch[J], AIEE Trans., (67), 1308-1313.

[6] Rabinow, J., 1951, Magnetic Fluid Torque and Force Transmitting Device [P], U.S.Patent.

[7] Chin, B.D., Park, J.H., Kwon, M.H., and Park, O.O., 1999, Rheological Properties and Dispersion Stability of Magnetorhelogical (MR) suspensions, Rheol. Acta., Vol.40, PP.211-219.

[8] Claracq, J., Sarrazin.J., and Montfort, J.P., 2004, Visco elastic properties of Magneto rhological fluids, Rheol, Acta, Vol 43, PP.38-49.

[9] Bica.I., 2006, Advances in Magnetorheological suspension: Production and Properties, J.Ind. Eng. Chem, Col 12, PP.501-515.

[10] Bombard, A.J.F., Knobel, M., and Alcantara, M.R., 2007, Phosphate coating on the surface of carbonyal Iron powder and its effect in Magneto rheological suspensions, Int.J.Modern Phys, B, Vol.21, PP 4858-4867.

[11] Pu, H.T., Jiang, F.J., and yang, Z.L., 2006, Preparation and properties of soft magnetic particles based on $\mathrm{Fe}_{3} \mathrm{O}_{4}$ and hollow Polystryrene microsphere composite, Matter. Chem. Phys., Vol.100, PP.10-14.

[12] Bossis, G., Khuzir, P., Lacis, S., and vol. Kova, O., 2003, Yield behaviour of magnetorheological suspensions, J.Magn Matter, Vol. 258-259, PP.456-458.

[13] MUHAMMAD Aslam., YAO Xiong-liang., an1d DENG Zhong-Chao., September 2006, Review of Magnetorheological (MR) Fluids and its Applications in vibration control, Journal of Marine Science and Application, Vol.5, No.3, PP:17-29.

[14] KORDONSKY.W.O., ASHOUR., ROGERS C.A., 1996, Maghetorheological fluids: Materials, characterization, and Devices [J]. Journal of Intelligent Material systems and structures (7), PP.123-130.

[15] Bia,I.,2006,AdvancesinMagnetorheologicalSuspension:Prod uctionandProperies,J.Ind.Eng.Chem,Vol.12.pp:501-515

[16] You, J.L., PARK, B.J., and Choi ${ }^{\mathrm{a}}$, H.J., 2007, Preparation and Magnetorheological Characterization of CI/PVB CORE/Shell Particle Suspended MR fluids, 2007, International Journal of Modern Physics B Vol.21, Nos 28 \& $294996-5002$.

[17] Min,S.,Cho.,Sung,T.,Lim.,Jang,In.Bb., Young,J.,Choi., and Myung.S.John.,JULY 2004,Encapsulation of Spherical Iron-Partile With PMMA and its Magnetorhological Particles, IE Transaction on Magnetics, Vol.40,No.4.

[18] Jolly, M.R., Bencler, J.W., and Calson, J.d., 1999, Properties 
and Applications of commercial Magnetorheological fluids J.Intell, Matter, Syst, Struct, Vol10, PP. 5-13.

[19] Choi, S.B., Song, H.J., Loo, H.H., Lim, S.C., Kim, J.H., and choi, H.J., 2003, vibration control of a Passenger volchel Featuring Magnetorheological Engine Mounts, Int.J.Vehicle Des., Vol.33. PP.2-16.

[20] Tran Hai Nam.,Kyoung Kwan Ahn.,and Jong 11 Yoon,2006,Proposition and Fabrication of a New Design of MR BrakeUsing the MR Fluid Proceedings of the KSME,Koreo,6(1)1263-1268.

[21] Tran Hai Nam.,Kyoung Kwan Ahn.,2007,Proposition and Fabrication MR Brake By Using the Combination of Conventional MR Fluid with ball bearing,Proceedings of the ICMT,The $11^{\text {th }}$ Interational Conferences on Mechatronics Technology Kores,27-46.

[22] Kim,J.H.,Oh,J,H.,2001,Development of an Above Knee Prosthesis Using MR Damper AND Leg Simulator ,Procedings of the JEEE,International Conference on Robotics and Automation,Seoul,Korea.

[23] Choi,J.S.,Park,b.j.,Cho.M.S.,and Choi,h.j.,2006,Preparation and Magnetorheological Characteristics of polymer Coated Carbonyl Iron Suspensins,Magnetism and Magnetic Materials,304,374-376.

[24] Park,b.j.,Jang,i.b.,Choi,H.J.,Pich,a.,Bhattacharya and Adler., 2006,Magnetorheological Characteristics of Nanoparticle -Added Carbonyl Iron System,Magnetism and Magnetic Materials303,290-293.

[25] Pu . T.H.JiangF,J.,and Yang , Z.I , 2006,preparation and properties of soft magnetic particles based on Fe3o4 and Hollow Polystyrene Microsphere Composite, Materials Chemistry and Physics 100 (1),10-14.

[26] Naayan,K.,Sudaa,A.,Yoshidaa,H.,Ohashib,.y.,Ogiwarab.h.,a nd Wakamatsu,r.,2007,MR fluid Viscous Coupling and its torque Delivery Control,Tribology International,89-97.

[27] Takesue.N.,Kiyota.y., and Furusho,j.,2002,Development of fast response MR-Fluid Actuatoe,SICE,02-0402.
[28] Yang,g.,Spencer,B,F.,Carlson.J.D and Sain,M.K.,2002,Large scale MR Fluid Dampers Modeling and Dynamic Performance Considerations,Engineering Stuctures 24 (3) $.309-323$

[29] Jung-Bac Jun.,Seong-Yong Uhm,-Hyun Ryu., and Kyung-Do Suh.,2005,Synthesis and characterization of Mondisperse Magnetic Composite Particles for Magnetorheological Fluid Materials, Colloids and Surfaces A:Physicocham Eng.Aspects 260,157-167.

[30] Kormann, C., Laun, H.M., and Richter, H.J., 1996 Int. J. Mod. Phys. B.10, 23.

[31] Wollny,k.,Lauger,j.,and Huck,s.,2002,Magnto Sweep-a New Method for Characterizing the Viscoelastic Properties for Magneto-rheological flui ds ,Appl.Rheol,Vol.12, pp:25-31.

[32] Jang, I.B., Kim, H.B., Lee, J.Y., You, J.L., Choi, H.J. and Jhon, M.S., 2005, Role of Organic coating on Carbonyl Iron suspended Particles in Magnetorheological Fluids,Journal of Applied Physics 97, 10Q912-1 to 10Q912-3.

[33] Gandhi,F.,andBullough,W.A.,2005,On the Pheno menological Modeling of Electro - rheologicaland Magne torheological FluidPreyield - Behavior,J.Int.Mater . Syst. Srruct, Vol.16,pp: 237- 247.

[34] Turczyn.R.,Kciuk.M.,April 2008,Preparation and Study of Model Magnetorheological Fluids, JAMME,Vol 27, issue 2.

[35] Bia,I.,2006,AdvancesinMagnetorheologicalSuspension:Prod uctionandProperies,J.Ind.Eng.Chem,Vol.12.pp:501-515.

[36] Bica.I. 2007, Giant Resistances Based on Manetorheological Suspension , j.Ind.Eng.Chem,Vol.13,pp: 299 304.1

[37] Client,E.,Maxey,R.M.,andKarniadakis,G.E.,2004,Dyanmics of Self Assembled Chaining in Magnetorheological Fluid ,Langmuir,Vol.20,pp:507-513.

[38] Bossis ,G.,Lemaire,E.,1991,Yeild Stress in Magnetic Sspension ,J.Rheol,vol.35,pp.1345-1354.

[39] Rann,P.J.,Horveth,A.T.,andKlingenberg.D.J.,1999,Magnetor heology in Viscoplatic Media,Rheol.acta,vol.38,pp:471-477. 